\title{
A numerical study of the effects of a Corotating Interaction Region on cosmic proton and helium transport
}

\author{
Xi Luo, ${ }^{a, *}$ Marius S Potgieter, ${ }^{b}$ Xueshang Feng, ${ }^{c}$ Fang Shen ${ }^{c}$ and Ming Zhang ${ }^{d}$ \\ ${ }^{a}$ Shandong Institute of Advanced Technology, Jingshi East Road, Jinan, 250100 China \\ ${ }^{b}$ Institute for Experimental and Applied Physics, CA University in Kiel, 24118 Kiel, Germany \\ ${ }^{c}$ National Space Science Center, Chinese Academy of Sciences, Beijing, 100190 China \\ ${ }^{d}$ Department of Physics and Space Sciences, Florida Institute of Technology, Melbourne, FL 32901 USA \\ E-mail:xi.luo@iat.cn
}

\begin{abstract}
A Corotating Interaction Region (CIR) is formed when the fast solar wind catches up with the slow solar wind. It is known that the intensity of Galactic Cosmic Rays (GCRs) is modulated by CIRs with the GCR intensity suppressed inside the CIR. Previous numerical studies were mainly confined to GCR protons. For this study we utilize a hybrid GCR transport model which incorporates a Magnetohydrodynamic (MHD) simulated solar wind plasma background with a CIR structure. Additionally, adopting appropriate mass, charge and local interstellar spectra, the hybrid transport model is applied to GCR protons and the two helium isotopes. It is found that both proton and total helium studied at $0.3 \mathrm{GV}$ are modulated by the CIR with their fluxes being depressed. However, the modulation for protons and helium is different with helio-longitude, and interestingly the ratio of the proton to helium flux varies with longitude. Similar results are found for the way in which the two helium isotopic fluxes vary.
\end{abstract}

$37^{\text {th }}$ International Cosmic Ray Conference (ICRC 2021)

July 12th - 23rd, 2021

Online - Berlin, Germany

\footnotetext{
*Presenter
} 


\section{Introduction}

It has been known for a long time that the speed of the solar wind which originate from a coronal hole is relatively fast. Caused by solar rotation, the solar wind stream forms a spiral structure and when the fast solar wind catches up with the preceding slow solar wind, the plasma between them is compressed and a Corotating Interaction Region (CIR) is formed.

Galactic Cosmic Rays (GCRs) penetrate into the heliosphere and when it interacts with the global solar wind and its imbedded magnetic field, solar modulation of these particle consequently follows, producing an 11-year solar cycle. CIRs also contribute to modulate GCR transport but on a different spatial and time scale. It is known that the solar rotation period of about 27 days is observed as recurrent variations in the GCR intensity originating from these CIRs [1]. Previous numerical studies about CIR effects on GCRs were mainly confined to cosmic protons e.g. [2, 3]. Here, we present a numerical model which is adapted to study the effect of a CIR on both proton and helium transport.

For a recent report of observed CIRs and their effects on cosmic rays, see e.g. [4] and references therein, and even at high helio-latitudes [5], and related studies [6].

\section{Numerical Model}

In this section, the numerical model for the current study is briefly discussed, specifically the background plasma model and the hybrid GCR transport model.

\subsection{Transport and Plasma Model}

\subsubsection{MHD model for inner heliosphere}

In this study, a sophisticated MagnetoHydroDynamic (MHD) numerical model has been utilized to model the plasma background in the inner heliosphere [8]. This model is based on the following idea MHD equation which in the conservation form gives:

$$
\begin{aligned}
\frac{\partial \rho}{\partial t}+\nabla \cdot(\rho \boldsymbol{V}) & =0, \\
\frac{\partial \rho \boldsymbol{V}}{\partial t}+\nabla \cdot\left[\left(P+\frac{B^{2}}{2 \mu_{0}}\right) \boldsymbol{I}+\rho \boldsymbol{V} \boldsymbol{V}-\frac{\boldsymbol{B} \boldsymbol{B}}{\mu_{0}}\right] & =-\frac{\rho G M_{s}}{r^{2}} \frac{\boldsymbol{r}}{r}+\boldsymbol{V} \cdot \boldsymbol{f}, \\
\frac{\partial \boldsymbol{B}}{\partial t}+\nabla \cdot(\boldsymbol{V} \boldsymbol{B}-\boldsymbol{B} \boldsymbol{V}) & =0, \\
\frac{\partial P}{\partial t}+\nabla \cdot(\rho \boldsymbol{V}) & =-(\gamma-1) P \nabla \cdot \boldsymbol{V} .
\end{aligned}
$$

Based on the total variation diminution Lax-Friedrich scheme and six-component mesh grid system, these four equations are solved in the simulation domain of a sphere with radius of 26 au. The simulated magnetic field and solar wind reflect the inner heliospheric plasma conditions. For a full report and detailed description, see Luo et al. [3]. 


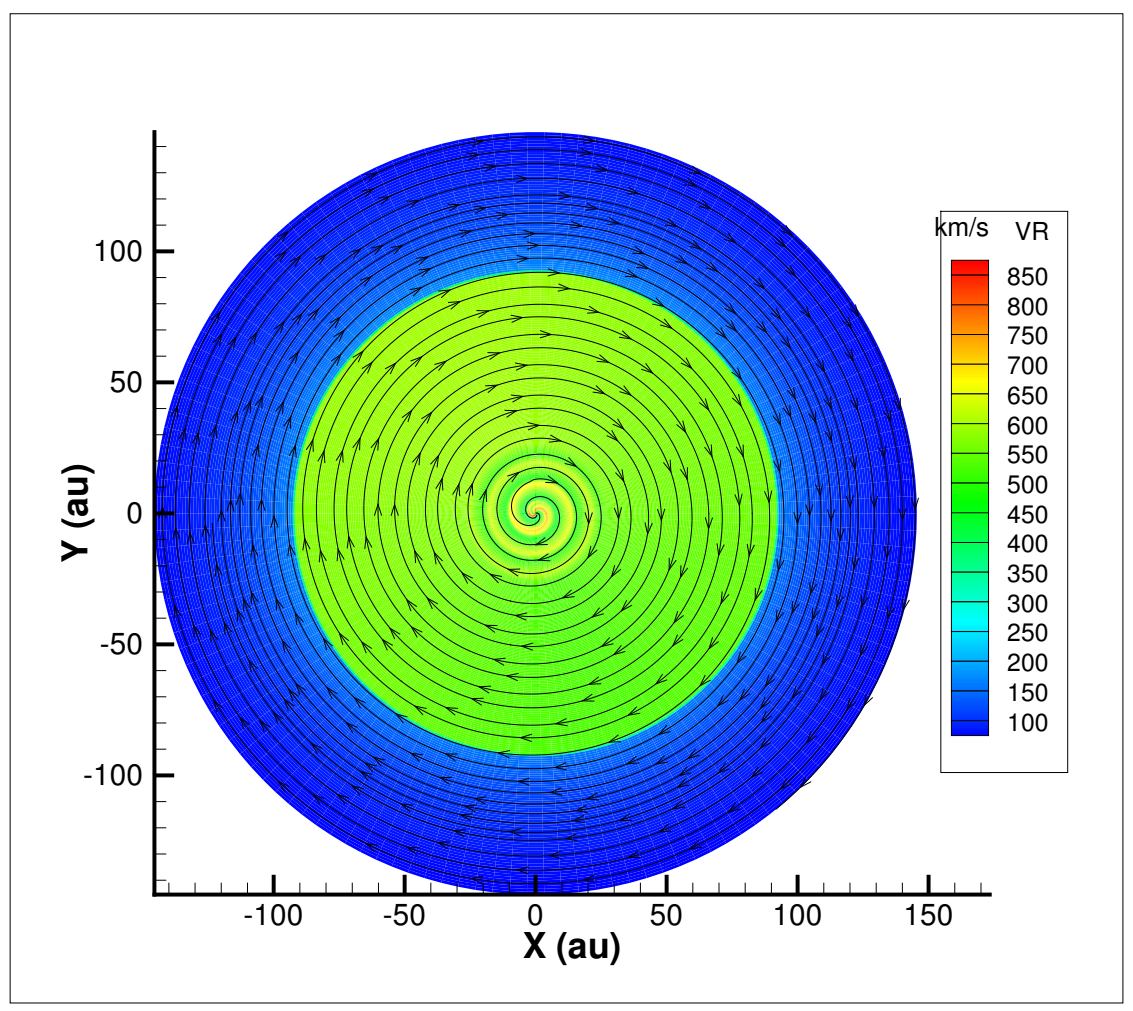

Figure 1: The heliosphere plasma profile (radial solar wind speed according to the colour coding scale on the right side and magnetic field spiral structure) in the ecliptic plane (XY-plane) combining the MHD simulation up to 26 au with Parker's IMF model in a sphere with a heliocentric radius of 130 au and a termination shock at $92 \mathrm{au}$, where both the magnetic field and solar wind change significantly.

\subsubsection{Parker's IMF model for the outer heliosphere}

It is known that GCRs orginate from regions far outside the solar system, and that their solar modulation inside the heliosphere is caused by the solar wind and its imbedded magnetic field, which applies to the whole heliosphere; see e.g. [9, 10]. It is therefore necessary to know the plasma information of the global heliosphere to model the transport process accurately. Since the MHD numerical model only provides the plasma background for the inner heliosphere up to 26 $\mathrm{au}$, we need an appropriate model for the rest of the heliosphere, adapting for this purpose Parker's Interplanetary Magnetic Field (IMF) model [11].

Figure 1 demonstrates the plasma profile, the radial solar wind and magnetic field in the ecliptic plane for the whole simulation domain. The profile for the inner heliosphere, with a heliocentric radius less than 26 au, is obtained from the MHD simulation, with Parker's IMF model for the rest of the heliosphere. This magnetic field at $(r, \theta, \phi)$ is connected with the boundary of the MHD simulated region at $\left(r_{0}, \theta_{0}, \phi_{0}\right)$ by the following formula:

$$
\begin{aligned}
& B_{r}(r, \theta, \phi)=B_{r}\left(r_{0}, \theta_{0}, \phi_{0}\right)\left(\frac{r_{0}}{r}\right)^{2}, \\
& B_{\theta}(r, \theta, \phi)=B_{\theta}\left(r_{0}, \theta_{0}, \phi_{0}\right), \\
& B_{\phi}(r, \theta, \phi)=B_{\phi}\left(r_{0}, \theta_{0}, \phi_{0}\right) \frac{r_{0}}{r} .
\end{aligned}
$$




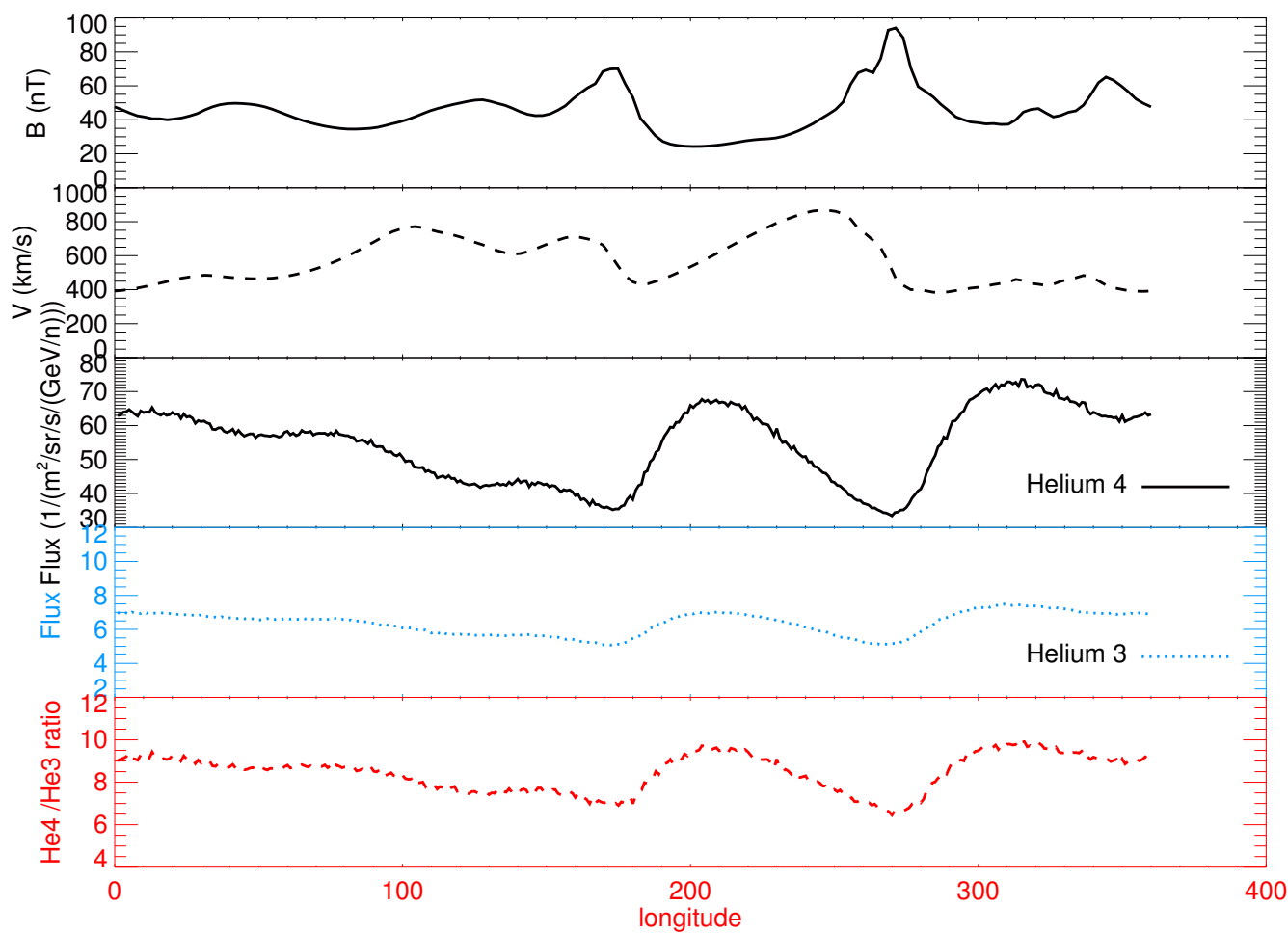

Figure 2: From the upper to lower panel, this figure shows the magnetic field magnitude $B$, solar wind speed $V$, He-4 flux, He-3 flux and the flux ratio of $\mathrm{He}-4$ to $\mathrm{He}-3$, as a function of helio-longitude at $0.3 \mathrm{GV}$. With the CIR structure steady during one solar rotation period, the flux variation with longitude reflects the recurrence of the GCR flux observed near the Earth during one solar rotation period.

Here $\left(r_{0}, \theta_{0}, \phi_{0}\right)$ is the "footpoint" position in the inner heliosphere boundary with radius $r_{0}$, by tracing the Parker spiral line from the point $(r, \theta, \phi)$. Correspondingly, the Parker spiral requires the following relationship for the polar angle $\theta_{0}$ and azimuthal angle $\phi_{0}$ :

$$
\begin{aligned}
\theta_{0} & =\theta \\
\phi_{0} & =\phi-\frac{r-r_{0}}{V_{s w}} \Omega \sin (\theta),
\end{aligned}
$$

where $\Omega$ is the angular speed of the solar rotation. The heliospheric termination shock is specified at a radius of 92 au where the magnetic field and solar wind speed both experience an abrupt change, as shown in Figure 1.

\subsection{Hybrid GCR transport model}

The GCR transport model is based on Parker's transport equation (TPE) [12] which can be cast into its Stochastic Differential Equation (SDE) forms; see [7, 13]:

$$
\begin{aligned}
d \vec{X} & =\left(\nabla \cdot K^{(s)}-\vec{V}_{s w}-\left\langle\overrightarrow{v_{D}}\right\rangle\right) d s+\sum_{\sigma} \overrightarrow{\alpha_{\sigma}} d W_{\sigma}(s), \\
d p & =\frac{1}{3} p\left(\nabla \cdot \vec{V}_{s w}\right) d s .
\end{aligned}
$$


The diffusion tensor $K^{(s)}$ and the drift velocity term $\left\langle\overrightarrow{v_{D}}\right\rangle$ is related to the interplanetary magnetic field $\vec{B}$, which is given by the plasma background model as mentioned. Similarly, the solar wind background profile is incorporated into the transport model; see [3].

The mentioned TPE describes four modulation processes for GCRs which are: convection caused by the outward flowing solar wind; diffusion because of scattering by the turbulent solar magnetic field; adiabatic energy losses determined by the divergence of solar wind velocity; drift of particles caused by magnetic field curvatures, gradients and the current sheet [9, 10, 15]. In 3D modeling there are three diffusion coefficients (DCs) of importance, one parallel to the background magnetic field direction, and two perpendicular to the field, respectively in the radial and polar directions [3, 9, 14].

Since GCR helium (total helium) is composed of helium-3 (He-3) and helium-4 (He-4) isotopes, which are modulated differently because of their mass-to-charge ratio, separate He-3 and He-4 very local interstellar spectra (LIS) from [14] are used in this numerical model, specified at the heliopause.

\section{Modelling results}

In this section, the initial results obtained from this hybrid numerical model are presented. Figure 2 displays how different entities vary with longitude. From the upper panel to lower panel, it demonstrates the magnetic field magnitude, solar wind speed, He-4 flux, He-3 flux and the flux ratio of He-4 to He-3. The CIR is a steady structure so that the variation with longitude actually represents how these quantities vary during one solar rotation period; see also [15]. In the MHD simulated plasma profile, the CIR is characterized by an enhanced magnetic field magnitude and steepening of the solar wind speed. As shown in the first panel, around $\phi=270^{\circ}$ and $\phi=170^{\circ}$, the CIR is showing its presence. Correspondingly, the He- 4 and He- 3 fluxes at $0.3 \mathrm{GV}$ generally decrease but with a variation in helio-longitude as shown in the third and fourth panels. Interestingly, the ratio of He-4 to He- 3 changes in the CIR region, as shown in the lower panel.

Figure 3, similar to Figure 2, displays how the proton and total helium fluxes vary as a function of helio-longitude. With the CIR showing it presence around $\phi=270^{\circ}$ and $\phi=170^{\circ}$, both helium and protons are modulated resulting in a decreased and varying flux. The computed ratio of the proton to helium fluxes, exhibits a similar behaviour, but less pronounced than for the He- 4 to He-3 ratio as shown in the previous figure.

\section{Discussion}

In this study, we have constructed a numerical model which is applied to investigate the effects of a CIR on the fluxes of GCRs, in particular for helium transport. This model is based on a MHD simulation, Parker's TPE and a SDE numerical method for solving it. The simulation reveals the basic features of the effects of a CIR on how the proton and helium fluxes vary with longitude where the CIR is present.

An interesting feature is that the flux ratio of $\mathrm{He}-4$ to $\mathrm{He}-3$ and proton to total $\mathrm{He}$ also vary with longitude due to the presence of the CIR. In this context, it is known that in the global heliosphere the proton to helium ratio varies at the Earth with time, from solar minimum to maximum, as reported for both PAMELA and AMS02; see e.g. [16, 17]. The reason for this global variation is 


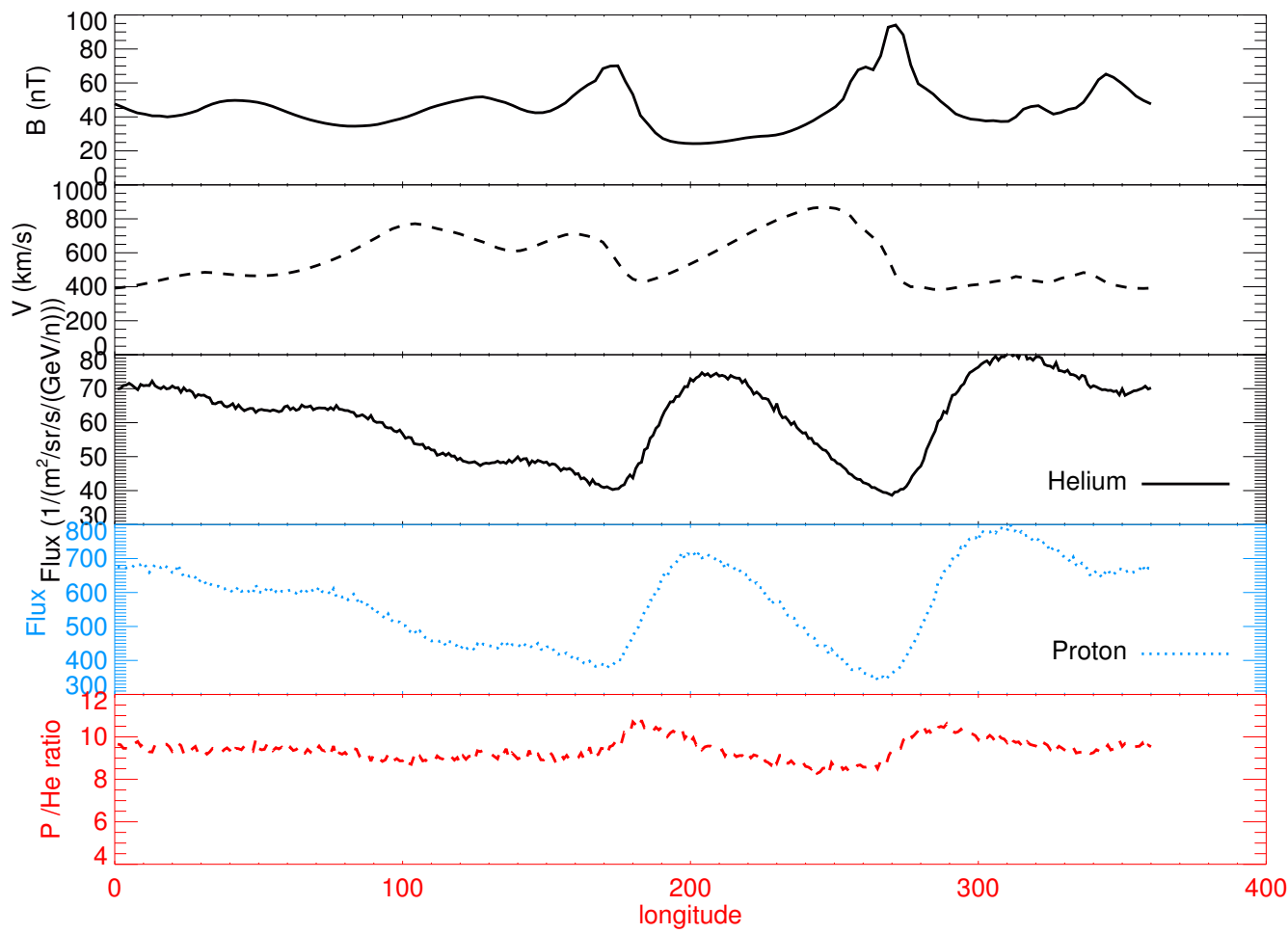

Figure 3: Similar to Figure 2, it displays how the proton and total helium flux, both at $0.3 \mathrm{GV}$, vary as a function of helio-longitude. In the bottom panel the corresponding proton to total helium flux ratio is shown.

not because there is a fundamental difference in their modulation but it lies in the fact that their very LIS are different in terms of spectral shape and at what rigidity the maximum flux (peak) occurs. When these different very LIS's are modulated from the heliopause to the Earth, the corresponding modulated spectra reiterate these differences. Apart from their very LIS, GCRs with different massto-charge ratios also respond differently to the modulation processes. The DCs for a $1 \mathrm{GV}$ proton are moderately different from the DCs for a He-3 and He-4 particle at 1 GV because the beta-factor (particle speed to speed of light) differs in the expressions for the DCs; the higher the rigidity, the less these differences become and the flux ratio becomes steady with time, only reflecting the ratio of the corresponding very LIS's. Obviously, the lower the rigidity, the larger the differences become so that the flux ratios become significantly larger with decreasing rigidity, down to where adiabatic energy losses shape the modulated spectra into having the same rigidity slopes, so that the flux ratios then do not change any more with further decreases in rigidity. See [14] for a detailed illustration of the differences in the very LIS's of these GCRs and how the flux ratios change with rigidity, according to a numerical modelling study, and [18, 19] for updates on this aspect of solar modulation.

Concerning the effects of a CIR on these particles with different mass-to-charge ratio, the first question that comes up is if the CIR in terms of modulation volume will have a similar additional effect on the flux ratio of these particles than in global modulation. It follows from our simulation that they generally do, and with a clear variation of the flux ratios with longitude, so that one has to look deeper into what is physically different inside a CIR causing these variations. Logically, 
because the magnetic field is enhanced and the solar wind speed is changed inside the CIR, both diffusion and convection as described in Parker's TPE are getting changed inside the CIR. In global modulation, changes in the DCs usually dominate the modulation process but when the solar wind speed is changed by a factor of two, changing the convection process has an impact. Evidently, it follows from Figure 2 that they both change by at least a factor of two inside the CIR. But, because charged particles respond to the magnetic field, not the solar wind, the diffusion process changing by a factor of two is dominant. The three DCs relevant here are normally of the form $\kappa=\kappa_{0} \beta f(\vec{r}) f(\vec{p})$, with the functional spatial dependence $f(\vec{r})$ usually assumed to scale as $1 / B$, with $B$ the magnitude of the magnetic field as shown in Figure 2, and with $\beta$ depending on the type of charged particles (nuclei and their isotopes). The functional rigidity dependence $f(\vec{p})$ is usually assumed to consist of the combination of two power laws, above around $4 \mathrm{GV}$ being different from below this value but these assumptions are identical for protons and helium; see e.g. [14]. This means that inside the CIR, these DCs change significantly and somewhat differently for the various nuclei and isotopes. Therefore, the CIR modulates proton, He-3 and He-4 also somewhat differently, all depending on what happens with $B$ and $V$ inside the CIR even if the GCRs have the same rigidity. This also implies that the CIR effects shown here should be much larger for GCR with lower rigidity; see [3-5]

Additionally, the drift coefficient, which always scales spatially as $1 / B$, also changes inside the CIR and can make a difference because particle drifts in the longitudinal direction may become meaningful if the CIR is getting large; for such effects, see e.g. [20,21] for 3D numerical studies of Forbush decreases for protons and electrons, and the corresponding charge-sign dependence. In this context, it will be worthwhile to study in detail the effects of a CIR on GCR electrons and compare it to that for protons and helium now that high precision and continuous observations of these GCRs are made simultaneously at the same place and at the same time; see [5] on what had been reported in this context for Ulysses observations.

\section{Summary and Conclusions}

We have found that: (1) GCR protons and the two helium isotopes studied at $0.3 \mathrm{GV}$ are modulated by the CIR so that their flux levels are depressed inside the CIR and in terms of how the fluxes vary with helio-longitude. (2) The manner in which the two helium isotopes respond to the presence of the CIR is somewhat different as a function of longitude. (3) Similarly, the manner in which protons and total helium respond to the presence of the CIR is different. (4) This gives the result that the flux ratio at $0.3 \mathrm{GV}$ of $\mathrm{He}-4$ to $\mathrm{He}-3$ and the ratio of protons to total helium vary inside the CIR; less for the proton to helium ratio than for the He-4 to He-3 ratio which is indicative of the difference in their respective very local interstellar spectra and the difference in their mass-to-charge ratio, a difference which dissipates at high rigidity but which becomes the dominant aspect with decreasing rigidity.

\section{References}

[1] J. A. Simpson, Space Sci. Rev. 83, 169, 1998.

[2] X. Guo and V. Florinski, Astrophys. J. 826, 65, 2016. 
[3] X. Luo, M. Zhang, X. Feng, et al., Astrophys. J. 899, 90, 2020.

[4] R. Modzelewska, G.A. Bazilevskaya, M. Boezio, et al., Astrophys. J. 904, 3, 2020.

[5] B. Heber, T. E. Sanderson and M. Zhang, Adv. Space Res. 23, 3, 567, 1999.

[6] M. Zhang, Astrophys. J. 488, 841, 1997.

[7] X. Luo, M. Zhang, K. H. Rassoul, et al., Astrophys. J. 764, 85, 2013.

[8] F. Shen, Z. Yang, J. Zhang, et al., Astrophys. J. 866, 18, 2018.

[9] M. S. Potgieter, Liv. Rev. Solar Phys. 10, 3, 2013.

[10] M. S. Potgieter, Adv. Space Res. 60, 848, 2017.

[11] E. N. Parker, Astrophys. J. 128, 664, 1958.

[12] E. N. Parker, Plant. Space Sci. 13, 9, 1965.

[13] M. Zhang, Astrophys. J. 513, 409, 1999.

[14] M. D. Ngobeni, O. P. M. Aslam, D. Bisschoff, et al., Astrophys. Space Sci. 365, 182, 2020.

[15] J. R. Jokipii and J. Kóta, J. Geophys. Res. Lett. 18, 1797, 1991.

[16] C. Corti, M. S. Potgieter, V. Bindi, et al., Astrophys. J. 871, 253, 2019.

[17] N. Marcelli, M. Boezio, A. Lenni, and PAMELA collaboration, Astrophys. J. 893, 145, 2020.

[18] M. D. Ngobeni, O. P. M. Aslam, D. Bisschoff, et al., this conference, 2021.

[19] M. D. Ngobeni, M. S. Potgieter, O. P. M Aslam, et al. this conference, 2021.

[20] X. Luo, M. S. Potgieter, M. Zhang and X. Feng, Astrophys. J. 839, 53, 2017.

[21] X. Luo, M. S. Potgieter, M. Zhang and X. Feng, Astrophys. J. 860, 160, 2018. 